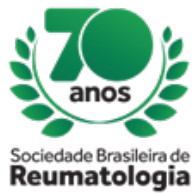

CENTRO DE EVENTOS DO CEARA O4 A 07 DE SETEMBRO

\title{
CYCLOPHOSPHAMIDE FOR TREATMENT OF DEMENTIA ASSOCIATED WITH ANTIPHOSPHOLIPID ANTIBODY SYNDROME: CASE REPORT
}

Mariana de Lira Fonte (Serviço de Clínica Médica - Hospital das Clínicas - Universidade Federal de Pernambuco, Recife, PE, Brasil), Marco Felipe Macêdo Alves (Serviço de Clínica Médica - Hospital das Clínicas - Universidade Federal do Pernambuco, Recife, PE, Brasil), Erick Barreto Pordeus (Serviço de Clínica Médica - Hospital das Clínicas - Universidade Federal do Pernambuco, Recife, PE, Brasil), Rebeca Cavalcanti Galle de Aguiar (Serviço de Clínica Médica - Hospital das Clínicas - Universidade Federal do Pernambuco, Recife, PE, Brasil), João Tavares Clemente Neto (Serviço de Clínica Médica - Hospital das

Clínicas - Universidade Federal do Pernambuco, Recife, PE, Brasil), Marília Rocha Costa (Serviço de Clínica Médica - Hospital das Clínicas - Universidade Federal do Pernambuco, Recife, PE, Brasil), Clezio Cordeiro de Sá Leitão (Serviço de Clínica Médica - Hospital das Clínicas - Universidade Federal de Pernambuco, Recife, PE, Brasil), Pedro Alves da Cruz Gouveia (Serviço de Clínica Médica - Hospital das Clínicas - Universidade Federal do Pernambuco, Recife, PE, Brasil)

\section{BACKGROUND}

Antiphospholipid syndrome (APS) is an autoimmune disorder characterized by thrombotic events and pregnancy morbidity in the presence of antiphospholipid antibodies. The APS can affect the central nervous system in different ways, with thrombotic events been related to most of manifestations. However, ischemic insults are not always enough to explain all clinical manifestations, so immunemediated damage has been advocated. Some degree of cognitive impairment is found in a high percentage of patients with primary APS, but the prevalence of dementia in these individuals is around 2.5\%. Improvement in cognitive function with use of anticoagulants has been reported, however, considering the mechanism of immune-mediated damage, immunosuppressive therapy can be considered. We report the case of a patient with dementia due to APS who underwent cyclophosphamide treatment because did not present improvement after one-year using anticoagulants.

\section{CASE REPORT}

A 46-year-old woman was admitted with a history of frequent and progressive forgetfulness with less than a year of evolution. She had difficulty performing basic activities of daily living and instrumental activities of daily living. She had a cranial computed tomography that showed more pronounced atrophy than expected for the age group, besides a focus of hypoatenuation of the left frontal region, probably related to previous ischemic insult. The patient also had a history of fetal loss around the 20th week of gestation. During the investigation, subacute deep vein thrombosis in the lower limbs was evident. With the possibility of thrombophilia, laboratory tests were requested for such investigation. High titles of anticardiolipin IgG and IgM and anti-beta-2 glycoprotein-I IgG and IgM were evidenced. In view of such findings, the diagnosis of APS was performed, being chosen for the indefinite use of warfarin and aspirin. A Mini-Mental State Examination (MMSE) was performed, with a score of 13 points, compatible with dementia. After about a year of outpatient follow-up, she did not present neurocognitive improvement even in the presence of anticoagulation. As a non-elderly patient with significant cognitive impairment, we chose to initiate monthly pulse therapy of cyclophosphamide for six months. In the fifth month of pulse therapy, a new MMSE was performed by the same evaluator, in which the patient had a score of 20 points, showing improvement in relation to the initial evaluation.

\section{CONCLUSION}

The use of cyclophosphamide is a therapeutic option in patients with APS dementia who do not present clinical improvement during anticoagulation. 